\title{
Pacific
}

Journal of

Mathematics

\section{STABLE CONSTANT MEAN CURVATURE SURFACES MINIMIZE AREA}

\author{
KARSTEN GROSSE-BRAUCKMANN
}




\title{
STABLE CONSTANT MEAN CURVATURE SURFACES MINIMIZE AREA
}

\author{
Karsten Grosse-Brauckmann
}

Let $M$ be an embedded strictly stable constant mean curvature surface, and $S$ a surface with the same boundary that encloses the same volume. If $S$ is sufficiently close to $M$ we show area $(S)>\operatorname{area}(M)$ unless $S=M$, i.e. $M$ is a local minimum of area.

R. Finn [F] has pointed out that for a general variational functional a positive second variation does not imply that an extremal is actually a local minimum, no matter if 'local' is taken in the $C^{0}, C^{1}$, or $C^{\infty}$ sense.

For minimal surfaces, however, it is known the above statement is true, see e.g. $[\mathrm{N}, \S 109]$. One way to prove this is to foliate a neighbourhood of a strictly stable extremal with minimal surfaces. The field of normals to the leaves is divergence-free, or a calibration, and the statement follows by integration.

There are two equivalent variational characterizations for a surface to have constant mean curvature $H$ : (i) it is an extremum of area under a volume constraint ( $H$ is the Lagrange parameter); or (ii) an extremum of area plus $H$ times the enclosed volume. If $H \neq 0$ they lead to different second variations (see $[\mathbf{B d C}]$ ). Positivity of the second variation for (i) is called strict volume preserving stability; for example this holds for any proper subset of the sphere. A stronger condition is that the second variation of (ii) is positive or strict stability; any proper subset of a hemisphere is strictly stable. We remark that spherical disks with the same boundary but varying mean curvature show that a strictly stable surface of constant mean curvature $H \neq 0$ is not a local minimum of area alone.

In $[\mathbf{W h}]$ it is shown that the functional (ii) is locally minimized by a strictly stable surface. As this proof uses regularity theorems from geometric measure theory we feel it is worth giving another proof that generalizes the idea for minimal surfaces mentioned before. This also gives for (i) that a strictly stable surface locally minimizes area among surfaces enclosing the same volume.

Under the weaker and physically more appropriate assumption of strict volume preserving stability it seems that local minimality for the functional (i) has not yet been addressed. Using an idea suggested by B. White we 
give a proof that shows local minimality for $C^{1}$-close graphs. The statement for arbitrary, merely $C^{0}$-close, surfaces then follows from the arguments of [Wh].

\section{Stability.}

We let $H=\operatorname{trace} A$ where $A$ is the second fundamental form of a hypersurface $M$ of $\mathbb{R}^{n+1}$. Then $S^{n}$ has mean curvature $H=n$ with respect to the inner normal. A hypersurface $M \subset \mathbb{R}^{n+1}$ of constant mean curvature $H$ is critical for the functional area $+H$ vol. One way to define the volume vol is to take a cone, $\operatorname{vol}(M)=\left(\int_{M} \nu(x) \cdot x\right) /(n+1)$, with $\nu$ the unit normal to $M$. Integration is always with respect to the volume element of $M$. We assume $M$ is compact and $C^{2}$, with $C^{2}$-boundary $\partial M$, and orientable if $H=0$.

The second variation of the functional (ii) on a surface of constant mean curvature $H$ depends only on the normal component $u$ of a variation field $X$,

$$
\text { (1) } \delta_{X}^{2}(\operatorname{area}(M)+H \operatorname{vol}(M))=-\int_{M} u \Delta u+|A|^{2} u^{2}=\int_{M}|D u|^{2}-|A|^{2} u^{2} \text {, }
$$

see e.g. [BdC, L.2.8]. Here we assumed $X$ (and $u$ ) to have compact support on $M ; \Delta$ is the Laplace-Beltrami operator of $M$. An immersed constant mean curvature surface $M$ is called stable iff

$$
\int_{M}-u \Delta u-|A|^{2} u^{2} \geq 0 \quad \text { for all } \quad u \in C_{c}^{\infty}(M),
$$

that is the lowest eigenvalue of $\Delta+|A|^{2}$ is nonnegative. If the lowest eigenvalue is positive we say the surface is strictly stable. Equivalently there is a $\lambda>0$ with

$$
\int_{M}-u \Delta u-|A|^{2} u^{2} \geq \lambda \int_{M} u^{2}
$$

If $H \neq 0$ then positivity of the second variation for (i) is equivalent to requiring (2) (resp. (3)) only for all $u$ with $\int_{M} u=0$, i.e. for those $u$ that do not change the volume; this is called (strict) volume preserving stability.

\section{Local minimality for strictly stable surfaces.}

For a strictly stable immersion $i_{0}: M \rightarrow \mathbb{R}^{n}$ of constant mean curvature $H_{0}$ we find a neighbourhood that is foliated by surfaces of the same constant mean curvature. Requiring that $i_{0}=\left.\mathrm{id}\right|_{M}$ we do not distinguish between $M$ and $i_{0}(M)$.

Theorem 1. Let $i_{0}: M \rightarrow \mathbb{R}^{n}$ be an immersed strictly stable hypersurface of constant mean curvature $H_{0}$ with $C^{2, \alpha}$ boundary $\partial M$. There exists a foliation 
$t \mapsto M_{t}$ of immersed surfaces with constant mean curvature $H_{0}$, that is an immersion $i: M \times[-1,1] \rightarrow \mathbb{R}^{n}$ with $i(M, 0)=i_{0}$ and $M_{t}=i(M, t)$. If $i_{0}$ is an embedding then $i$ is a diffeomorphism onto its image.

Proof. (See [MY, Thm. 3], [N, §414] for the proof for minimal surfaces.) We consider normal perturbations of $M$ of the form $i_{0}(x)+u(x) \nu(x)$, where $\nu$ is the normal to $M$ and $u$ is in the Banach space $C^{2, \alpha}(\bar{M})$. Then $i_{0}+$ $u \nu$ is a $C^{2, \alpha}$-immersion if $u$ has sufficiently small $C^{2, \alpha}$-norm, in particular the mean curvature function $H\left(i_{0}+u \nu\right)$ is defined. We now want to use the implicit function theorem for the map $\sigma$ from $C^{2, \alpha}(\bar{M})$ to $C^{0, \alpha}(\bar{M}) \times$ $C^{2, \alpha}(\partial M)$ defined by $u \mapsto\left(H\left(i_{0}+u \nu\right)-H_{0},\left.u\right|_{\partial M}\right)$.

The linearization of $\sigma$ at $u \equiv 0$ is given by

$$
d \sigma(v)=\left(\Delta v+|A|^{2} v,\left.v\right|_{\partial M}\right)
$$

(see [K, App.C], note that $H$ is the first variation of area, and $\int d H(v) v$ is the second variation of area). By strict stability the only solution $v$ to the equation $d \sigma(v)=(0,0)$ is $v \equiv 0$. The Fredholm alternative for weak solutions [GT, Thm. 8.6] therefore gives a unique solution $v \in W^{1,2}(\bar{M})$ to the equation $d \sigma(v)=h$ with $\left.v\right|_{\partial M}=\varphi$ for each $h \in L^{2}$ and $\varphi \in C^{2, \alpha}(\partial M)$. Moreover if $h \in C^{0, \alpha}(\bar{M})$ then by [GT, Thm. 8.29] $v \in C^{0, \alpha}(\bar{M})$. The Schauder estimates yield $\|v\|_{C^{2, \alpha}(\bar{M})} \leq C\left(\|v\|_{C^{0, \alpha}(\bar{M})}+\|h\|_{C^{0, \alpha}(\bar{M})}+\|\varphi\|_{C^{2, \alpha}(\partial M)}\right)$ for $h=\left(\Delta+|A|^{2}\right) v$ with a constant $C$ depending on the coefficients, see [GT, Thm. 6.6]. This shows that for $h \in C^{0, \alpha}$ the weak solution $v$ is in fact in $C^{2, \alpha}(\bar{M})$. We conclude that $d \sigma$ is a diffeomorphism between the Hölder spaces.

The inverse function theorem for Banach spaces then gives a neighbourhood of $(0,0) \in C^{0, \alpha}(\bar{M}) \times C^{2, \alpha}(\partial M)$ such that $\sigma$ is invertible. In particular there are preimages $u_{t} \in C^{2, \alpha}(\bar{M})$ of the constants $(0, t)$ for $t$ sufficiently small. After scaling in $t$ as necessary we let $i(M, t)$ be the map $i_{0}+u_{t} \nu$.

It remains to show $i(M, t)$ is an immersion. Choosing $t$ smaller if necessary (resp. scaling in $t$ ) it is sufficient to prove that $v:=d u_{t} / d t$ is positive. Now $v$ is a solution to the linearized equation $L v=\Delta v+|A|^{2} v=0$ on $M$ with boundary values $(d / d t) t=1$. By assumption the first eigenvalue of $L$ on $M$ is positive. The statement follows from the next lemma.

Usually, for the maximum principle it is required that the coefficient of the term linear in the function be negative (see, e.g. [GT, Cor. 3.2]). This condition can be relaxed up to a positive number less than the first eigenvalue, see [H, Problem on p. 92]. We include a proof as we are not aware of a reference.

Lemma 2. Let $L v=a^{i j} D_{i j} v+b^{i} D_{i} v+c v$ be a linear elliptic equation with 
smooth coefficients. Suppose that its first eigenvalue on a bounded $C^{2, \alpha}$ domain $\Omega \subset \mathbb{R}^{n}$ is positive. If $L v \leq 0$ and $\inf _{\partial \Omega} v>0$ then $\inf _{\Omega} v>0$.

Proof. The minimum of $v$ cannot be 0 since then $v \equiv 0$ by the Hopf maximum principle, see the remark following Thm. 3.5 of [GT].

We now suppose that $v$ takes a negative minimum. Since $\Omega$ has $C^{2, \alpha}$ boundary we can extend the coefficients smoothly to a neighbourhood of $\Omega$. We let $\tilde{\Omega}$ be a neighbourhood of $\Omega$ so that the extended operator still has a positive first eigenvalue. Let $\xi$ be the first eigenfunction on $\tilde{\Omega}$, that is $L \xi+\lambda \xi=0$ with $\lambda>0$ and $\left.\xi\right|_{\Omega}>0$. Writing $v=\xi w$ on $\Omega$ we find that $w$ also takes a negative minimum at some point $y$. Since $\left.v\right|_{\partial \Omega}>0$ the point is in the interior, $y \in \Omega$, and $D w(y)=0, a^{i j} D_{i j} w(y) \geq 0$. Hence at $y$

$$
\begin{aligned}
0 & \geq L v=L(\xi w) \\
& =a^{i j} D_{i j} \xi w+b^{i} D_{i} \xi w+c \xi w+\xi a^{i j} D_{i j} w+\xi b^{i} D_{i} w+2 a^{i j} D_{i} \xi D_{j} w \\
& =(L \xi) w+\xi a^{i j} D_{i j} w \\
& \geq-\lambda \xi w .
\end{aligned}
$$

We conclude $w(y) \geq 0$ and $v(y) \geq 0$ in contradiction to our assumption.

Using the foliation we prove the local minimality for the functional (i) assuming its second variation is positive. We let $i: M \times[-1,1] \rightarrow \mathbb{R}^{n}$ be a foliated embedding by surfaces with constant mean curvature $H$, and let $N$ be its image.

Theorem 3. Let $M$ be a strictly stable embedded surface of constant mean curvature $H$ and $S$ any $C^{1}$-surface with $\partial S=\partial M$ that is contained in the foliation $N=i(M \times(-1,1))$ defined in Theorem 1 . Then either area $(S)+$ $H \operatorname{vol}(S)>\operatorname{area}(M)+H \operatorname{vol}(M)$ or $S=M$. In particular, if $S$ and $M$ enclose the same volume then either $S=M$ or $\operatorname{area}(S)>\operatorname{area}(M)$.

Proof. We consider the unit vector field $\nu$ on $N$ normal to each leaf $M_{t}$ so that $\left.\nu\right|_{M_{-1}}$ is the interior normal to $N$. The divergence of $\nu$ on a leaf $M_{t}$ is given by $\operatorname{div}_{M_{t}} \nu=\sum_{i} e_{i} \cdot D_{e_{i}} \nu=-H$, where $e_{i}$ is an orthonormal basis to the tangent space of $M_{t}$. From $\nu \cdot \nu=1$ we get $\nu \cdot D_{\nu} \nu=0$ and hence the divergence with respect to $N$ is also given by $\operatorname{div} \nu=-H$.

Now $-H \operatorname{vol}(S)=\int_{V_{S}} \operatorname{div} \nu$ and by the divergence theorem this is equal to $\int_{S} \nu \cdot \xi-\int_{M_{-1}} \nu \cdot \nu$, where $\xi$ is the unit normal to $S$ that agrees with the exterior normal to $V_{S}$. A similar formula holds for $M$ and we obtain $-H \operatorname{vol}(S)+H \operatorname{vol}(M)=\int_{S} \nu \cdot \xi-\int_{M} \nu \cdot \nu \leq \operatorname{area}(S)$ - area $(M)$ with strict inequality unless $S$ is tangential to the foliation in all points. Thus either $\operatorname{area}(M)+H \operatorname{vol}(M)<\operatorname{area}(S)+H \operatorname{vol}(S)$ or $S=M$. 
We thank Rob Kusner for the communication of a similar idea of proof; it is used by Rossman [R] to simplify the proof of the following result of Meeks [M, Sect. 4]: If there is a foliation with leaves $M_{s}$ of constant mean curvature $H_{s}$ then leaves that are critical for the mean curvature are stable. Wente used a similar calibration argument for capillary surfaces [W].

Clearly our statements generalize to embedded constant mean curvature hypersurfaces in orientable $n$-manifolds.

\section{Local minimality for volume preserving stable surfaces.}

We now address the problem of minimality of area with a volume constraint under the (weaker) assumption of volume preserving stability. The main difficulty is that the constraint $\operatorname{vol}\left(i_{0}+u \nu\right)=0$ is non-linear, that is it does not imply $\operatorname{vol}\left(i_{0}+t u \nu\right)=0$. Following an idea of Brian White, for given $M$ we replace area with a volume constraint by the functional

$$
\mathcal{F}_{C}=\operatorname{area}+H \operatorname{vol}+\frac{C}{2}[\operatorname{vol}-\operatorname{vol}(M)]^{2} .
$$

The first variation of $[\operatorname{vol}-\operatorname{vol}(M)]^{2}$ vanishes evaluated at $M$. Therefore critical points of the functionals area $+H$ vol and $\mathcal{F}_{C}$ agree:

Lemma 4. Let $C \in \mathbb{R}$. Then $M$ has constant mean curvature $H$ iff $M$ is critical for $\mathcal{F}_{C}$.

For a volume preserving stable surface the second variation of $\mathcal{F}_{C}$ is positive for all test functions, not just for the volume preserving ones provided we choose $C$ big enough.

Lemma 5. Let $\lambda$ be a positive number such that $\delta_{u \nu}^{2}($ area $+H$ vol $) \geq \lambda \int u^{2}$ holds for all $u \in C_{c}^{\infty}(M)$ satisfying the volume constraint $\int u=0$. Then there is a $C>0$ such that $\delta_{u \nu}^{2} \mathcal{F}_{C} \geq \lambda \int u^{2}$ holds for all $u \in C_{c}^{\infty}(M)$.

Proof. The second variation of $\mathcal{F}_{C}$ is $\delta^{2} \mathcal{F}_{C}=\delta^{2}($ area $+H$ vol $)+C(\delta \text { vol })^{2}$. Hence by (1)

$$
\delta_{u \nu}^{2} \mathcal{F}_{C}=\int_{M}|D u|^{2}-|A|^{2} u^{2}+C\left(\int_{M} u\right)^{2}
$$

Suppose the statement does not hold. Then for each $n \in \mathrm{N}$ in place of $C$ there is a function $u_{n}$ with $\delta_{u_{n} \nu}^{2} \mathcal{F}_{n}<\lambda \int_{M} u_{n}^{2}$, or

$$
\int_{M}\left|D u_{n}\right|^{2}-|A|^{2} u_{n}^{2}+n\left(\int_{M} u_{n}\right)^{2}<\lambda \int_{M} u_{n}^{2} .
$$


In particular $\int\left|D u_{n}\right|^{2}<\int\left(|A|^{2}+\lambda\right) u_{n}^{2}<$ const $\left\|u_{n}\right\|_{L^{2}}^{2}$. Further assuming $u_{n}$ is normalized with $\left\|u_{n}\right\|_{L^{2}}=1$ we obtain that the $W^{1,2}$-norm of the $u_{n}$ is bounded. Hence $u_{n}$ converges weakly in $W^{1,2}$, and by Rellich's theorem strongly in $L^{2}$, to a function $u$. For the left hand side of (5) to be finite, $u$ must satisfy the volume constraint $\int u=0$ and so (5) gives $\int|D u|^{2}-$ $|A|^{2} u^{2}<\lambda \int u^{2}$, in contradiction to our assumption.

We can now prove the main statement for graphs.

Theorem 6. Let $i_{0}: M \rightarrow \mathbb{R}^{3}$ be an immersed volume preserving stable surface of constant mean curvature $H$. Then there is a constant $c$ as follows: If a graph over $M$ is of the form $i_{0}+u \nu$, with $\|u\|_{C^{0,1}}<c$ and $\left.u\right|_{\partial M}=0$, and it encloses the same volume, then it has bigger area than $M$ unless $u \equiv 0$.

Proof. For $u$ with sufficiently small $C^{0,1}$-norm we aim at an estimate

$$
\mathcal{F}_{C}\left(i_{0}+u \nu\right)=\mathcal{F}_{C}\left(i_{0}\right)+\delta_{u \nu}^{2} \mathcal{F}_{C}\left(i_{0}\right)+o\left(\|u\|_{L^{2}}^{2}+\|D u\|_{L^{2}}^{2}\right) .
$$

A computation leading to (3.23) of [BT] gives

(7)

$$
\begin{aligned}
& \operatorname{area}\left(i_{0}+u \nu\right)+H \operatorname{vol}\left(i_{0}+u \nu\right) \\
& =\operatorname{area}\left(i_{0}\right)+H \operatorname{vol}\left(i_{0}\right)+\int_{M}\left(|D u|^{2}-|A|^{2} u^{2}\right)+o\left(\|u\|_{L^{2}}^{2}+\|D u\|_{L^{2}}^{2}\right) .
\end{aligned}
$$

A similar formula for the volume (same page of $[\mathbf{B T}]$ ) states

$$
\operatorname{vol}\left(i_{0}+u \nu\right)-\operatorname{vol}\left(i_{0}\right)=\int_{M} u-\int_{M} \frac{H}{2} u^{2}+\int_{M} \frac{K}{3} u^{3},
$$

where $K$ is Gauß curvature of $M$. By the assumption on the enclosed volume the left hand side vanishes and $\int u \leq \sup _{M}(|H| / 2+|u||K| / 3)\|u\|_{L^{2}}^{2} \leq$ const $\|u\|_{L^{2}}^{2}$, using that the $C^{0}$-norm of $u$ is bounded. Squaring the last equation gives

$$
\left(\int_{M} u\right)^{2}=o\left(\|u\|_{L^{2}}^{2}\right)
$$

Now the estimate (6) follows from (7) and (4).

On the other hand, by the preceding lemma we have strict stability for $\mathcal{F}_{C}$ with sufficiently large $C$, namely $\int|D u|^{2}-|A|^{2} u^{2}+C\left(\int u\right)^{2} \geq \int \lambda u^{2}$ for all $u$. Multiplying with $(1-\varepsilon)$ we get

$$
\begin{aligned}
& \int_{M}(1-\varepsilon)|D u|^{2}-|A|^{2} u^{2}+(1-\varepsilon) C\left(\int_{M} u\right)^{2} \\
& \geq \int_{M}\left[(1-\varepsilon) \lambda-\varepsilon|A|^{2}\right] u^{2} \geq \tilde{\varepsilon} \int_{M} u^{2}
\end{aligned}
$$


where $\tilde{\varepsilon}$ is a lower estimate for [...]; we choose $\varepsilon>0$ small enough so that $\tilde{\varepsilon}$ is positive. Adding $\varepsilon \int|D u|^{2}$ to both sides we obtain with a new constant $\mu=\min (\varepsilon, \tilde{\varepsilon})$

$$
\delta_{u \nu}^{2} \mathcal{F}_{C}\left(i_{0}\right) \geq \mu\left(\|u\|_{L^{2}}^{2}+\|D u\|_{L^{2}}^{2}\right)
$$

We now combine (6) and (9) to obtain

$$
\mathcal{F}_{C}\left(i_{0}+u \nu\right) \geq \mathcal{F}_{C}\left(i_{0}\right)+\frac{\mu}{2}\left(\|u\|_{L^{2}}^{2}+\|D u\|_{L^{2}}^{2}\right)
$$

for all $u$ with sufficiently small $C^{0,1}$-norm. In particular if $\mathcal{F}_{C}\left(i_{0}+u \nu\right) \leq$ $\mathcal{F}_{C}\left(i_{0}\right)$ then $u \equiv \mathbf{0}$.

The theorem generalizes to hypersurfaces of $\mathbb{R}^{n}$ since (8) clearly extends to this case. The graph hypothesis in the preceding theorem can be removed with some regularity results. Indeed using Lemma 5 this follows from Theorem 3 of $[\mathbf{W h}]$.

Added in proof: F. Tomi kindly pointed out the following reference to us. H. Ruchert: Ein Eindeutigkeitssatz f̈̈ Flächen konstanter mittlerer Krümmung (Arch. Math., 33 (1979), 91-104). Therein our Theorem 1 and 3 are proven on the assumption that $M$ is a disk-type immersed $C_{2}$-surface contained in an open ball of radius $2 / H$ with $\partial M$ real analytic.

\section{References}

[BdC] J. Barbosa and M. do Carmo, Stability of hypersurfaces with constant mean curvature, Math. Z., 185 (1984), 339-353.

[BT] R. Böhme and F. Tomi, Zur Struktur der Lösungsmenge des Plateauproblems, Math. Z., 133 (1973), 1-29.

[F] R. Finn, Editor's note, to appear Pac. J.

[GT] D. Gilbarg and N.S. Trudinger, Elliptic partial differential equations of second order, Second edition, Springer, 1983.

[H] G. Hellwig, Partial differential equations, Second edition, Teubner, 1977.

[K] N. Kapouleas, Complete constant mean curvature surfaces in Euclidean three-space, Ann. of Math., 131 (1990), 239-330.

[M] W.H. Meeks, The topology and geometry of embedded surfaces of constant mean curvature, J. Diff. Geom., 27 (1988), 539-552.

[MY] W.H. Meeks and S.T. Yau, The existence of embedded minimal surfaces and the problem of uniqueness, Math. Z., 179 (1982), 131-168.

[N] J.C.C. Nitsche, Lectures on minimal surfaces I, Cambridge University Press, 1989.

[R] W. Rossman, Thesis, Univ. of Mass., 1992. 
[W] H. Wente, Stability for the axially symmetric pendent drop, Preprint, MPI Bonn, 1987.

[Wh] B. White, A strong minimax property of nondegenerate minimal submanifolds, J. Reine Ang. Math., 457 (1994), 203-218.

Mathematisches Institut

UNIVERSITÄT BONN

BERINGSTR. 1

53115 Bonn, GERManY

E-mail address: kgb@rhein.iam.uni-bonn.de 
Mogens L. Hansen and Richard V. Kadison, Banach algebras with uni-

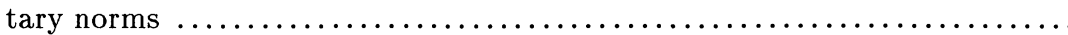

Xin-hou Hua, Sharing values and a problem due to C.C. Yang ..........

Jing-Song Huang, Harmonic analysis on compact polar homogeneous

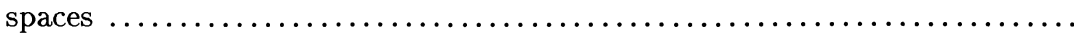

Min-Jei Huang, Commutators and invariant domains for Schrödinger prop-

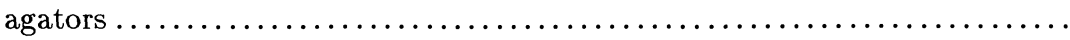

Hisao Kato, Chaos of continuum-wise expansive homeomorphisms and dy-

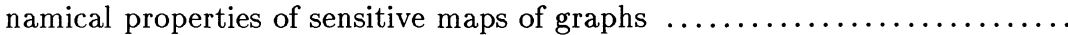

Oliver Küchle, Some properties of Fano manifolds that are zeros of sections in homogeneous vector bundles over Grassmannians ...................

Xin Li and Francisco Marcellan, On polynomials orthogonal with respect to Sobolev inner product on the unit circle $\ldots \ldots \ldots \ldots \ldots \ldots \ldots \ldots \ldots \ldots$. . . . . . .

Steven Liedahl, Maximal subfields of $Q(i)$-division rings .............. 147

Alan L.T. Paterson, Virtual diagonals and $n$-amenability for Banach algebras ....................................................

Claude Schochet, Rational Pontryagin classes, local representations, and

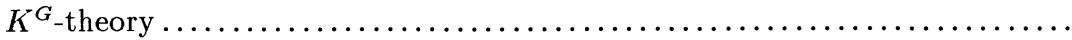

Sandra L. Shields, An equivalence relation for codimension one foliations of 3-manifolds

D. Siegel and E. O. Talvila, Uniqueness for the $n$-dimensional half space

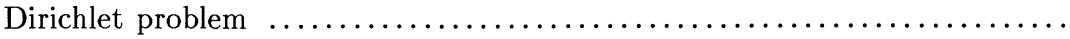

Aleksander Simonič, A Construction of Lomonosov functions and applica-

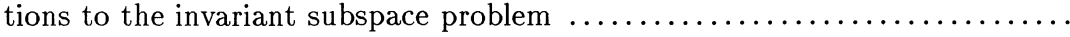

Endre Szabó, Complete intersection subvarieties of general hypersurfaces.. 


\section{PACIFIC JOURNAL OF MATHEMATICS}

Volume $175 \quad$ No. $2 \quad$ October 1996

Mean-value characterization of pluriharmonic and separately harmonic functions

LeV Abramovich AĬZEnberg, CARlos A. Berenstein and L.

WERTHEIM

Convergence for Yamabe metrics of positive scalar curvature with integral bounds on 307 curvature

\section{KAZUO AKUTAGAWA}

Generalized modular symbols and relative Lie algebra cohomology

\section{AVNER DOLNICK ASH and DAVID GINZBURG}

Convolution and limit theorems for conditionally free random variables

MaReK BożejKo, Michael LEINERT and Roland SPEICHER

$L^{p}$-bounds for hypersingular integral operators along curves

SHARAD CHANDARANA

On spectra of simple random walks on one-relator groups. With an appendix by Paul Jolissain

Pierre-Alain Cherix, Alain J. Valette and Paul Jolissaint

Every stationary polyhedral set in $\mathbf{R}^{n}$ is area minimizing under diffeomorphisms

JAIGYOUNG CHOE

Ramanujan's master theorem for symmetric cones

Hongming Ding, Kenneth I. GRoss and Donald Richards

On norms of trigonometric polynomials on SU(2)

AnTHONy H. DOOLEY and SANJiV Kumar GuPTA

On the symmetric square. Unit elements

YUVAL ZVI FLICKER

Stable constant mean curvature surfaces minimize area

KARSTEN GROSSE-BRAUCKMANN

Banach algebras with unitary norms

Mogens LEMVIG HANSEN and RiCHARD VinCENT KADISON

Harmonic analysis on compact polar homogeneous spaces

JING-SONG HUANG

Uniqueness for the $n$-dimensional half space Dirichlet problem

DAVID SIEGEL and ERIK O. TALVILA 